\title{
DEFINIÇÕES FORMAIS DE MOBILIDADE E ACESSIBILIDADE APOIADAS NA TEORIA DE SISTEMAS DE MARIO BUNGE
}

\author{
MAGALHÃES, M. T. Q. \\ ARAGÃO, J. J. G. \\ YAMASHITA, Y.
}

\section{RESUMO}

Sobre o tema da mobilidade e acessibilidade, o foco dos pesquisadores tem estado demasiadamente voltado para aplicações práticas sem, contudo, atentar em desenvolver a teoria subjacente, resultando em conceitos inflados por definições confusas e vagas. Em meio a diversas acepções existentes para os termos Mobilidade e Acessibilidade, muita confusão emerge. Neste contexto, o presente trabalho propõe a formalização de uma definição para os dois conceitos, com vistas a retirar a ambiguidade inerente à linguagem natural. Partindo da teoria de Mario Bunge e da reflexão sobre a natureza do Transporte, são propostas definições gerais formalizadas para as noções de acessibilidade e mobilidade, o que traz uma série de implicações para a pesquisa e desenvolvimento metodológico na área.

Palavras-Chave: Teoria de Sistemas, Mobilidade, Acessibilidade, Conceito, Definição, Formalização.

\section{ABSTRACT}

On the subject of mobility and accessibility, most researchers have been focused on practical applications, without giving proper attention to the development of the underlying theory, resulting in conceptual inflation caused by confusing and vague definitions. Among the existing definitions for terms such as Mobility and Accessibility, confusion emerges. This paper aims to formalize such notions and try to remove the ambiguity that is inherited from the natural language. We use the theory of Mario Bunge and the discution on the Nature of Transport to propose general formal definitions for Accessibility and Mobility.

Keywords: Systems Theory, Mobility, Accessibility, Concept, Definition, Formalization. 


\section{INTRODUÇÃO}

Os termos acessibilidade e mobilidade têm recebido tantas definições quantos são os autores que abordam o tema. Contudo, diante desta miríade de definições, emerge mais confusão que clareza, o que dificulta demasiadamente o trabalho dos pesquisadores. Grande parte desta confusão resulta da dificuldade em se perceber qual o conteúdo inovador ou distintivo oferecido por um ou outro autor. Ou ainda, que a linguagem utilizada - a linguagem natural - é demasiadamente ambígua. Outro fato interessante é que muitas das definições descuidam com a etimologia dos termos acessibilidade e mobilidade, o que não é indicado, mesmo quando se está tratando de uma definição especializada. Atribuímos isso ao foco dos pesquisadores demasiadamente voltado para aplicações práticas sem, contudo, atentar em desenvolver a teoria subjacente.

As noções de acessibilidade e mobilidade são centrais no campo de planejamento de transporte e/ou da mobilidade urbana e, portanto, merecem um cuidadoso trabalho em sua definição, de forma a dotá-los de valor operacional para o desenvolvimento do campo de investigação. Neste sentido, o ideal de formalização pode ser assumido como um desafio para os pesquisadores e pensadores da área, de forma a reduzir a ambiguidade relacionada à interpretação do vocábulo.

Coloca-se, assim, a questão: tendo em vista a etimologia dos termos acessibilidade e mobilidade, como formalizar estes dois termos, ainda levando em consideração noções como sistemas de transporte e transporte? Que teoria e que linguagem poderiam oferecer suporte a tal formalização?

Neste artigo, busca-se a definição formalizada e integrada para as noções de mobilidade e acessibilidade, inclusive tendo como pano de fundo a noção de transporte e sistema de transporte. Aborda-se, portanto, um aspecto de desenvolvimento que tem sido negligenciado pela pesquisa na área. Neste sentido, far-se-á uso da Teoria de Sistemas de Mario Bunge, com sua linguagem específica e notações utilizadas em lógica simbólica, inclusive a lógica modal.

O trabalho está estruturado em 6 seções, além da introdução. Na seção 2, são apresentadas e discutidas algumas das principais e mais adotadas definições de acessibilidade e mobilidade. Após isso, na seção 3, são apresentados os principais pontos da Teoria de Sistemas de Bunge, que servem de base para a formação das noções em pauta. Em seguida, discute-se o conceito de transporte e sistema de transporte, e apresenta-se as noções de mobilidade e acessibilidade sob o quadro teórico apresentado. Na seção 5, são oferecidas definições formalizadas para acessibilidade e mobilidade. Por fim, conclui-se com comentários gerais sobre as implicações das definições propostas e indicativos de novos estudos e desenvolvimentos.

\section{PRINCIPAIS ACEPÇÕES DOS TERMOS ACESSIBILIDADE E MOBILIDADE}

O termo acessibilidade tem assumido diferentes acepções dependendo do autor que o define. Etimologicamente, acessibilidade deriva do latim accessiblitas, que significa "livre acesso, possibilidade de aproximação" (Houaiss, 2001). Contudo, raramente os autores tendem a partir do sentido original do termo. Como exceção, temos Girle (2003, p.36) que, 
ao tratar da metafísica dos mundos possíveis, define acessibilidade como uma relação, e para clarificar a ideia, sugere uma descrição de "poder ver" ou "ser visto". Em outras palavras, nesta acepção, se algo é acessível, ou dotado de acessibilidade, é possível o estabelecimento de uma relação entre esse algo e outra coisa qualquer.

Especificamente na área de Transporte e Mobilidade diversas definições estão disponíveis, a exemplo:

- Cox (1972, p.176) diz que "Acessibilidade contém duas importantes noções: aquela de ser capaz de alcançar um lugar - a componente de conexão da acessibilidade; e aquela de ser capaz de chegar lá de forma rápida e/ou barata - a componente de custos de movimento da acessibilidade."

- Taaffe et al. (1996, p.12) de forma semelhante a Cox (1972) considera acessibilidade como facilidade de acesso de um ponto aos demais pontos de uma rede.

- Vuchic (2000, p.199) define acessibilidade como a "habilidade de viajar entre diferentes atividades".

- Hansen apud Fotheringham et al (2000) define acessibilidade como uma medida derivada do número de oportunidades e da distância.

- Ortúzar \& Willumsen (1994) definem acessibilidade como a facilidade (ou dificuldade) de realizar viagens de/para uma determinada zona de tráfego.

- Lei 10.098/2000 (Brasil, 2000) define acessibilidade como possibilidade e condição de alcance para utilização, com segurança e autonomia, dos espaços, mobiliários e equipamentos urbanos, das edificações, dos transportes e dos sistemas e meios de comunicação, por pessoa portadora de deficiência ou com mobilidade reduzida.

- Lei 12.587/12 estabelece como acessibilidade facilidade disponibilizada às pessoas que possibilite a todos autonomia nos deslocamentos desejados.

Percebe-se que algumas definições muito fogem da noção original do termo, muitas vezes confundindo a ideia com suas condições de realização. Isto, no âmbito da pesquisa, provoca muitas confusões entre os pesquisadores. Nossa preocupação aqui é a proposição de uma definição geral de mobilidade e acessibilidade que permita sua especificação a casos especializados, sem, contudo, "inflar" demasiadamente esses conceitos, fato que vem ocorrendo paulatinamente.

\section{Mobilidade: algumas acepções}

Inicialmente, cabe trazer à tona, sua acepção no senso comum. Etimologicamente, o termo "mobilidade" deriva do latim, mobilitas(átis), que por sua vez deriva de mobilis(e) que significa móvel (que pode se mover). Neste sentido, que domina o senso comum, mobilidade é entendida como a característica do que é móvel, do que tem a capacidade de se deslocar. Assim, é a própria capacidade de mudança, de deslocamento, capacidade esta própria de uma entidade.

Nesta acepção, o termo mobilidade foi apropriado e delimitado por diversas áreas. A sociologia, a eletrônica, a ciência da computação, a física, a geografia (demografia) e pela área de Planejamento Urbano e de Transportes. Cada uma considera a mobilidade como um fenômeno diferenciado, de acordo com a abordagem de cada ciência, e para isto, utiliza de distintas definições. A Definição é de suma importância na prática científica, já que, dentre outras coisas, elimina a ambigüidade, aclara significados e explica teoricamente os objetos (Copi, 1978). 
A ETC/CDS (apud EEA, 2006), por exemplo, define como mobilidade "a capacidade de grupos ou indivíduos de mudar de residência, de trabalho ou de se mover fisicamente de um lugar para outro".

O Ministério das Cidades (2004), por sua vez, coloca que a mobilidade abrange um conjunto de políticas de transporte, circulação, acessibilidade e trânsito. E, assim posta, não explica muito do conceito.

Recentemente, a Lei 12.587/2012 (Brasil, 2012) definiu como mobilidade urbana como condição em que se realizam os deslocamentos de pessoas e cargas no espaço urbano;

As definições acima colocadas trazem significados distintos para a mobilidade. Assim como no caso da acessibilidade, buscamos aqui uma definição formalizada geral. Para isso, é necessário utilizar uma base teórica para a construção coerente destas definições.

\section{BASE TEÓRICO-FILOSÓFICA: BUNGE E A VISÃO DE SISTEMA}

Em discurso não-formalizado, Bunge (1979, p.4) afirma que qualquer sistema tem composição definida, ambiente definido e estrutura definida. A composição do sistema é o conjunto de seus componentes; o ambiente, o conjunto de itens com os quais ele está conectado; e a estrutura, as relações entre seus componentes, bem como entre estes e os elementos do ambiente.

O autor também apresenta uma distinção entre agregado e sistema. Para Bunge (1979, p.4), agregado é

(...) uma coleção de itens sem qualquer vinculo entre eles, e portanto, sem integridade e unidade. Agregados podem ser tanto conceituais quanto concretos (materiais). Um agregado conceitual é um conjunto (mas nem todo conjunto é um agregado conceitual: um conjunto dotado de uma estrutura é um sistema conceitual). Um agregado concreto ou material, por outro lado, é uma coisa composta, cujos componentes não estão acoplados, ligados, conectados ou vinculados, tal qual um campo constituído por dois outros campos superpostos (...)

e sistema é

(...) um objeto complexo, cujos componentes são inter-relacionados. Se os componentes são conceituais, assim também é o sistema; se são concretos ou materiais, então eles constituem um sistema concreto ou material. Uma teoria é um sistema conceitual, uma escola é um sistema concreto do tipo social.

A primeira formalização do conceito de sistema conforme proposto por Bunge (1979, p.5) é como segue. Seja $T$ um conjunto não-vazio. Então, o terno ordenado $\sigma=\left\langle\mathscr{C}, \mathscr{E}, \mathscr{Q}^{\prime}\right\rangle$ é (ou representa) um sistema sobre $T$, se $\mathscr{C}$ e $\mathscr{E}$ são subconjuntos mutuamente excludentes de $T$ (ex. $\mathscr{C} \cap \mathscr{E}=\varnothing$ ), e $\mathscr{Q}$ é um conjunto não-vazio de relações na união de $\mathscr{C}$ e $\mathscr{E}$. O sistema é conceitual se $T$ é um conjunto de itens conceituais, e concreto (ou material) se $T \subseteq \Theta$ é um conjunto de entidades concretas, por exemplo coisas (para a definição precisa de coisa, ver Bunge, 1977, cap. 1 a 3).

Entretanto, segundo o autor, para se obter a definição adequada, as noções de composição $(C)$, ambiente (E) e estrutura (S) precisam ser formalizadas. Essas formalizações, conforme 
propostas por Bunge, são apresentadas a seguir, especificamente para os sistemas concretos.

\section{Definição de sistema concreto por Bunge}

Para compreender a definição de sistema dada por Bunge, é necessário ter as definições de A-composição, A-ambiente e A-estrutura - esclarecendo que o ' $\mathrm{A}$ ' corresponde ao vocábulo 'atômico'. Neste texto, as notações serão as mesmas utilizadas no texto original de Bunge.

Conforme entende o autor, a composição de um sistema não é apenas uma coleção de suas partes, mas o conjunto de seus "átomos". E define, simbolicamente, a composição absoluta e a A-composição da seguinte forma (Bunge, 1979, p.5-6):

Seja $A \subseteq \Theta$ uma classe de coisas e $x$ uma coisa qualquer (ex. $x \in \Theta$ ). A composição (absoluta) de $x$ é o conjunto de suas partes:

$$
\mathscr{C}(x)=\{y \in \Theta \mid y \sqsubset x\}
$$

onde:

$$
\begin{aligned}
& \mathscr{O}(x) \text { : Composição absoluta de } x \\
& \text { ' } y \sqsubset x \text { ' designa " } y \text { é parte de } x \text { " }
\end{aligned}
$$

E a A-composição de $x$ é o conjunto de suas A-partes (coisas do tipo 'A'):

$$
\mathscr{C}_{A}(x)=\mathscr{\mathscr { C }}(x) \cap A=\{y \in A \mid y \sqsubset x\}
$$

onde:

$$
\mathscr{F}_{A}(x): \text { A-composição de } x
$$

Necessário à compreensão de seu modelo, Bunge (1979, p.6) introduz o conceito de ligação, conexão ou acoplamento entre componentes de uma coisa. Para ele, esse conceito - de conexão - é distinto de relações como "ser mais velho", "estar ao lado" etc. Duas coisas estão conectadas quando pelo menos uma delas age sobre a outra. E, se uma coisa age sobre outra, ela modifica a linha de comportamento, trajetória ou história da última. A relação de ação é simbolizada da seguinte forma: se uma coisa $a$ age sobre uma coisa $b$ escreve-se que ' $a \triangleright b^{\prime}$.

A noção de A-ambiente de uma coisa $x$ com uma A-composição $\mathscr{C}_{A}(x)$ é definida como o conjunto de todas as coisas, que não estão contidas em $\mathscr{C}_{A}(x)$, que agem sobre, ou recebem a ação de $\mathscr{C}_{A}(x)$. Na linguagem formal adotada por Bunge, escreve-se:

$$
\mathscr{E}_{A}(x)=\left\{y \in \Theta I \neg\left(y \in \mathscr{\mathscr { C }}_{A}(x)\right) \&(\exists x)(z \sqsubset x \&(y \triangleright z \vee z \triangleright y))\right\} \text { (3) }
$$

onde

$$
\begin{aligned}
& \mathscr{E}_{A}(x) \text { : A-Ambiente de } x \\
& \mathscr{C}_{A}(x) \text { : A-Composição de } x
\end{aligned}
$$


Por fim, a estrutura é o conjunto de todas as relações entre os componentes de uma coisa, bem como as relações entre estas e as coisas no ambiente. A formalização da definição de estrutura, para um sistema concreto, é apresentada no próximo item.

\section{Formalização das características de sistema concreto por Bunge}

Um objeto é um sistema concreto se, e somente se, for composto por pelo menos duas coisas diferentes e conectadas (Bunge, 1979, p.6).

As três características de um sistema, cujas noções foram adiantadas no subitem anterior, são (Bunge, 1979, p.7): A-Composição, A-Ambiente e A-Estrutura.

Seja $\sigma \in \sum$ um sistema concreto e $A \subseteq \Theta$ uma classe de coisas. A A-composição de $\sigma$ no tempo $t$ é o conjunto de todas as suas A-partes em $t$. Na linguagem formal de Bunge escreve-se:

$$
\mathscr{C}_{A}(\sigma, t)=\{x \in A \mid x \sqsubset \sigma\}
$$

O A-ambiente de $\sigma$ no tempo $t$ é o conjunto de todas as coisas do tipo $A$, que não são componentes de $x_{1}$ mas que agem ou sofrem a ação de componentes de $\sigma$ no tempo $t$. Em termos formais da linguagem utilizada por Bunge, escreve-se como segue:

$$
\left.\mathscr{E}_{A}(\sigma, t)=\left\{x \in A \mid x \notin \mathscr{\mathscr { C }}_{A}(\sigma, t) \&(\exists y)\left(y \in \mathscr{C}_{A}(\sigma, t)\right) \&(x \triangleright y \vee y \triangleright x)\right]\right\}
$$

O A-estrutura de $\sigma$ no tempo $t$ é o conjunto de relações, em particular conexões, entre os componentes de $\sigma$, e entre estes e as coisas no A-ambiente de $\sigma$ no tempo $t$. Em termos formais da linguagem utilizada por Bunge, escreve-se da seguinte forma:

$$
\mathscr{Q}_{A}(\sigma, t)=\left\{R_{i} \in \mathbb{B}_{A}(\sigma, t) \cup \overline{\mathbb{B}}_{A}(\sigma, t) \mid \mathbb{B}_{A}(\sigma, t) \neq \varnothing \& 1 \leq i \leq n\right\}
$$

Onde:

$\mathbb{B}_{A}(\sigma, t):$ conjunto de conexões

$\overline{\mathbb{B}}_{A}(\sigma, t)$ : conjunto de relações que não são de conexão

$R_{i: \text { uma relação qualquer }}$

\section{DEFININDO ACESSIBILIDADE E MOBILIDADE A PARTIR DA DEFINIÇÃO DE TRANSPORTE}

\section{Levantamento de aspectos ligados ao vocábulo 'transportar'}

O vocábulo transportar pode assumir as seguintes acepções, estas selecionadas em função da relevância para a discussão (Houaiss et al., 2001):

- (transitivo direto, bitransitivo) levar ou conduzir (seres animados ou coisas) a (determinado lugar); carregar;

- (pronominal) passar-se ou mudar-se de um lugar para outro, transferir-se.

A partir disso, chama-se atenção para alguns aspectos do verbo transportar: primeiro, suas acepções aqui apresentadas referem-se a mudança de lugar; segundo, no processo, existe 
um objeto (seres animados ou coisas); e terceiro, pode assumir forma reflexiva, indicando que quem transporta pode ser também o que é transportado. Esses aspectos serão importantes para a discussão do conceito de transporte realizado mais adiante.

Ainda com relação ao vocábulo, cabe observar que ele pode assumir forma substantiva, transporte, designando, assim, uma classe específica de fenômenos.

\section{Levantamento de definições de Transporte em manuais especializados}

Os paradigmas sintetizam os principais elementos de uma área de estudos, contendo elementos ontológicos, teóricos, metodológicos (Magalhães, 2010). Os paradigmas de um campo de estudos podem ser identificados nos manuais utilizados para a formação dos novos pesquisadores. Assim, a consulta a esses manuais pode oferecer uma definição mais especializada do termo transporte, enriquecendo, assim, a reflexão sobre a natureza do fenômeno.

Morlock (1978, p.5) traz duas acepções para a palavra "transporte" tiradas de dicionários de língua inglesa: (i) an act, process, or instance of transporting or being transported; $e$, (ii) to transfer or convey from one place to another.

As duas acepções apresentadas assemelham-se àquelas apresentadas na seção anterior, incluindo a forma reflexiva (transportar e ser transportado) observada. Pode-se observar, entretanto, que a primeira definição apresentada por Morlock faz uma referência circular, não explicando o que é transportar. Esse tipo de definição é muito comum em dicionários e é aceito quando se trata de linguagens naturais. Entretanto, pouco tem a oferecer em contextos mais especializados. Sua importância, no máximo, reside em noções sintáticas sobre o vocábulo.

Ainda sobre Morlock (1978, p.5), ele traz a definição de engenharia de transportes como sendo

a aplicação de ciência e matemática pela qual as propriedades da matéria e das fontes de energia na natureza são utilizadas para movimentar passageiros e mercadorias de forma útil à humanidade.

Antes de discutir a definição dada por Morlock, cabe reproduzir a definição trazida por Papacostas e Prevedouros (1993, p.1) para sistema de transporte como sendo

as instalações fixas, as entidades de fluxo, e o sistema de controle que permite que pessoas e mercadorias vençam a fricção do espaço geográfico de forma eficiente, permitindo participar tempestivamente em alguma atividade desejada.

Nas definições acima, um aspecto em especial é digno de atenção: a noção de que o transporte serve a um propósito, um desejo (expresso em "useful to mankind" $e$ "in order to participate in a timely manner in some desired activity"), ou seja, tem como uma de suas propriedades a intencionalidade.

\section{Transporte e ação intencional}

O Transporte é um processo humano intencional e algumas das definições vistas anteriormente sugerem esta noção. Os fenômenos percebidos no dia-a-dia como as pessoas se deslocando de um lado para outro, veículos automotores lotando ruas e uma 
infinidade de outros exemplos são apenas uma parte desse processo mais complexo, cuja totalidade não é observável se limitada a esta gama de "aparências". Para melhor compreender isso, tome-se a analogia feita por Andler et al. $(2005$, p.567) para explicar a dificuldade de observação da ação intencional:

Imaginemos o caso de Pedro cruzando na rua com a diretora da escola primária que seus filhos frequentaram. Ele tinha, na época, brigado com ela e se pergunta se não deveria propor uma reconciliação. O que ele imagina, depois decide fazer, não é deslocar seu braço e sua mão, de modo que seu chapéu seja brevemente levantado, em cinco centímetros acima de sua cabeça; também não é dar, a um fotógrafo de emboscada, a oportunidade de ilustrar um artigo sobre os costumes antiquados de uma cidadezinha do interior;(...) o que Pedro faz, deliberadamente (...), é cumprimentar a diretora da escola que seus filhos frequentaram. $O$ agente escolhe fazer $X$, e realiza sua escolha fazendo $Y$ : o primeiro "fazer" tem sentido, aparentemente, diferente do segundo - no caso mais simples, $X$ é realizado pelo agente, ao passo que Y só é realizado, de fato, por uma parte do corpo do agente.

Em relação ao Transporte vale a analogia. Os fenômenos observados e comumente chamados de Transporte são apenas parte da ação intencional. Esta envolve a escolha do sujeito $(X)$, a qual é realizada fazendo-se, dentre outras coisas, o deslocamento de um objeto (Y), por exemplo: (1) o produtor de soja do Mato Grosso quer vender seu produto na Europa, ele realiza essa ação exportando o produto através de um porto no Maranhão; ou (2) um estudante deseja participar das atividades educacionais, ele realiza essa ação deslocando-se até a escola na qual está matriculado. Cabe observar que seria possível citar uma série de ações na categoria Y. Entretanto, o ponto destacado aqui é a percepção de que o processo de transporte envolve duas dimensões: uma explícita e observável que não é a determinante, mas apenas a manifestação de uma escolha; e, uma velada, a ação intencional, engajada, que é a determinante. Ou seja, a real razão pelo qual os deslocamentos observados ocorrem não é conhecida senão pelo próprio "Sujeito de Transporte". Qualquer observador exterior pode apenas conjecturar sobre as razões, ou então questionar diretamente o agente.

Analisando o fenômeno Transporte: um modelo

Para analisar os fenômenos de transporte, postule-se que existe um processo de transporte e que é composto por alguns momentos distintos, conforme descrito abaixo:

- Momento 01: Uma pessoa (ou grupo de pessoas) precisa desenvolver alguma atividade com vistas a satisfazer alguma necessidade. Ela sabe, ou acredita, com base no senso comum ou outra forma de conhecimento, que a viabilização de sua participação em uma atividade implica uma série de ações concatenadas, dentre as quais inclui aquelas que resultarão no deslocamento de um objeto material específico (objeto tem sentido sintático, ou seja, ele "sofre" a ação);

- Momento 02: Ela procura as formas que dispõe para realização das diversas ações, notadamente aquelas que promoverão esse deslocamento;

- Momento o3: Caso exista alguma forma que atenda às suas expectativas, ela decide por acionar ou não os recursos que dispõe;

- Momento o4: Uma vez acionados, os meios realizam o deslocamento segundo os requisitos postos pela pessoa; 
- Momento 05: O deslocamento é finalizado. A pessoa (ou grupo de pessoas) pode desenvolver a atividade que desejava e satisfazer sua expectativa.

Isto posto, o processo de transporte pode ser apresentado conforme Figura 01:

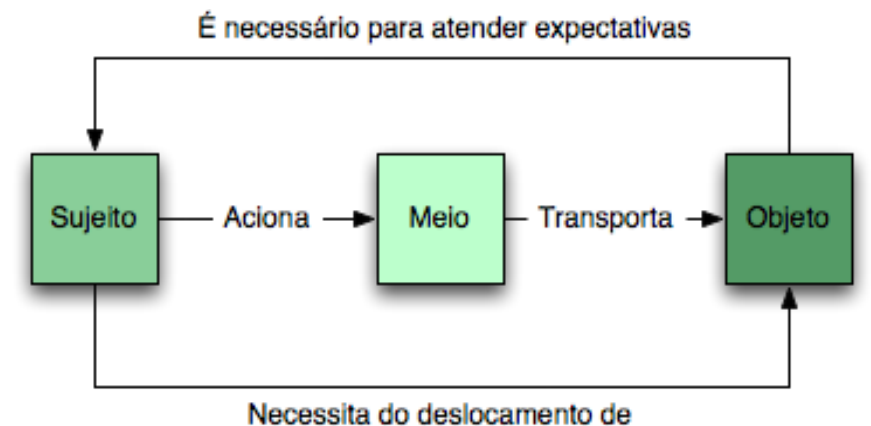

Fig. 1. Elementos determinantes do fenômeno de Transporte

Ou seja, o transporte depende de três elementos para a sua realização: o sujeito do transporte, o meio de transporte e o objeto do transporte. O Sujeito do Transporte é aquele que possui alguma necessidade ou desejo cuja satisfação requer o deslocamento de um objeto qualquer. O Objeto do Transporte, por sua vez, é aquilo cujo deslocamento é necessário para a satisfação das expectativas do Sujeito de Transporte. Para exemplificar, tome-se uma fábrica que deseja produzir seus produtos e para isso precisa que insumos sejam levados até sua unidade produtiva. A fábrica é o Sujeito de Transporte, e o insumo, o Objeto.

No que diz respeito a uma ação específica de transportar, a relação entre o Sujeito e o Objeto é mediada por um outro ente, o Meio de Transporte. O Meio de Transporte é aquilo que efetivamente transporta o objeto. Continuando o exemplo anterior, a fábrica pode não ser a responsável pelo deslocamento, podendo contratar, por exemplo, um serviço que se responsabilize por apanhar o produto onde quer que ele esteja, e entregá-lo no local desejado pela fábrica. O Meio de Transporte é, assim, o responsável efetivo pelo fenômeno que observamos de um objeto mudar de um lugar para o outro.

O esquema apresentado pode, por vezes, conduzir a entendimentos equivocados e deve-se ter atenção. Para ilustrar, cabe citar dois casos exemplares:

Situação 01: Uma indústria de automóveis contrata o transporte de seus produtos a uma concessionária situada em outra região geográfica.

- Sujeito do Transporte: a indústria de automóveis;

- Meio de Transporte: o sistema que envolve a infraestrutura de transporte existente (veículos, vias, edificações, equipamentos), o operador do serviço, dentre outros agentes;

- Objeto do Transporte: os automóveis.

Situação 02: Um estudante desloca-se a pé para a escola.

- Sujeito do Transporte: o estudante;

- Meio de Transporte: o sistema que envolve a infraestrutura de transporte existente (calçadas, calçadões, passarelas e outros espaços do pedestre), e parte do corpo do estudante (seu sistema locomotor);

- Objeto do Transporte: o estudante (especificamente, seu corpo). 


\section{A ACESSIBILIDADE E A MOBILIDADE DEFINIDAS NO CONTEXTO DO TRANSPORTE}

A interação entre três elementos é fundamental para a produção do fenômeno de transporte: o Sujeito do Transporte, o Meio do Transporte e o Objeto do Transporte. Para que o fenômeno do transporte possa acontecer, uma relação deve ser estabelecida entre o Sujeito e o Meio e entre o Meio e o Objeto, no sentido do transporte. Como foi visto, a relação no sentido sujeito-meio é 'aciona' e no sentido meio-objeto é 'transporta'. O resultado da possibilidade de estabelecimento dessas relações determina uma propriedade ao objeto: a mobilidade, a propriedade daquilo que pode ser transportado. A acessibilidade é, por definição, uma propriedade do meio do transporte que pode interagir com o sujeito e com o objeto, no âmbito específico do transporte. Desta forma, a acessibilidade pode ser decomposta em dois componentes: a acessibilidade sujeito-meio e a acessibilidade meioobjeto.

Morfossintaticamente, os vocábulos 'acessibilidade' e 'mobilidade' são utilizados para designar propriedades. Partindo disso como postulado, e explorando os esquemas anteriormente apresentados, partimos para a reflexão sobre a natureza dessas propriedades e qual a característica dos atributos para representá-las.

Podemos dizer que tanto a propriedade de acessibilidade (atribuída, no nosso caso específico, a sistemas de transporte apenas), quanto de mobilidade (atribuída exclusivamente a objetos), são propriedades mútuas, não-intrínsecas - sobre isso, ver Magalhães (2010). Ou seja, os atributos que as representam são, necessariamente, de aridade maior que 1 - por exemplo, uma coisa é acessivel a outra ( $x A y$ ou $A x y$ ).

Sobre a aridade do atributo da mobilidade como sendo de ordem maior que 1 , Morlok (1978, p.83) oferece um bom exemplo:"(...) objeto se move em algum tipo de veículo, o qual dá ao objeto mobilidade num tipo particular de caminho utilizado(...)".

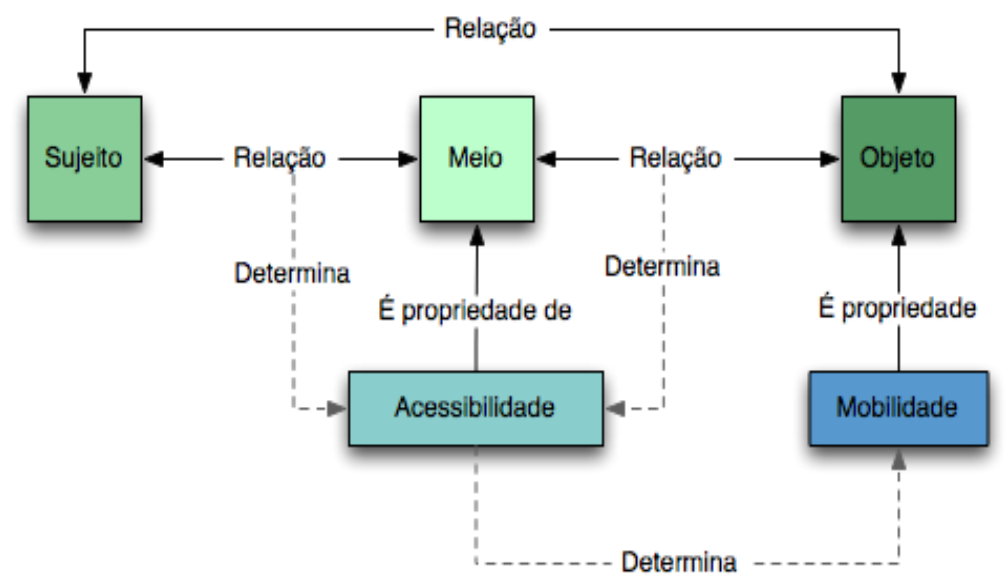

Fig. 2. Relações entre os elementos fundamentais do Transporte e as propriedades fundamentais do Meio e do Objeto de Transporte

Ter isso em mente é de fundamental importância se almejamos a formalização desses conceitos. Muitas definições apresentadas para esses dois termos tem sido ambíguas e teoricamente equivocadas. Aqui, fornecemos uma definição geral de acessibilidade. 


\section{Formalização Geral da Acessibilidade}

Seja $C \subset \mathscr{\mathscr { C }}_{A}(\sigma, t), E \subset \mathscr{E}_{A}(\sigma, t), S \subset \mathscr{Q}_{A}(\sigma, t), r \in S$, uma relação $r: \mathscr{E}_{A}(\sigma, t) \rightarrow \mathscr{C}_{A}(\sigma, t)$ pertencente à A-Estrutura de um sistema de transporte $\sigma \in \Sigma$. Sejam ainda $x \in E$ e $y \in C$.

Diz-se que $x$ tem acesso ao sistema $\sigma$, ou " $A_{\sigma} x$ ", se, e somente se, existe um elemento $x$ do A-Ambiente do sistema que estabelece uma relação com um elemento $y$ (distinto do primeiro) da A-Composição do sistema. Isso pode ser formalizado da seguinte forma:

$$
A_{\sigma} x \leftrightarrow \exists x \exists y \exists r(x \in E \wedge y \in C \wedge \diamond\langle x, y\rangle \in r)
$$

O sinal gráfico ' $\diamond$ ' é o operador modal de possibilidade. Para maiores detalhes sobre a notação, sintaxe e semântica, sistemas e propriedades específicas da lógica modal consultar Hughes e Cresswell (1996).

Por exemplo, seja $x$ uma indústria de alimentos, $y$ uma empresa de transportes de cargas (que compõe o sistema de transporte de cargas), e $r$ uma relação " $x$ contrata e paga $y$ ". Dizemos que, nesse caso, o sistema de transporte de cargas é acessível à indústria de alimentos se, e somente se, for possível que a empresa contrate e pague o valor cobrado pela empresa transportadora (ex. existem escritórios da empresa na localidade, ou meios de comunicação para estabelecimento do contato, a indústria dispõe de recursos financeiros suficientes, etc).

Observe que, neste momento, estamos preocupados com a definição geral de acessibilidade e não nas condições específicas sob as quais as relações podem ser estabelecidas, devendo este aspecto ser objeto de outras investigações e trabalhos científicos.

\section{Formalização Geral da Mobilidade}

A formalização da Mobilidade depende das noções estabelecidas anteriormente para Acessibilidade. Mas antes de partir para a formalização propriamente dita, façamos uma digressão sobre a ideia de mobilidade.

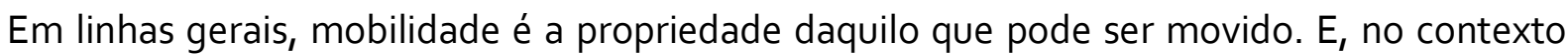
do sistema de transporte, para se dizer que algum objeto ou pessoa tem a mobilidade como propriedade, deve existir um sistema de transporte que possa ser acionado e que possa receber, transportar e entregar o objeto. Ou seja, conforme a Figura 2, um objeto é móvel (possui a propriedade mobilidade) se, e somente se, o sistema de transporte é acessível ao sujeito de transporte e ao objeto de transporte.

Formalizando a ideia chegamos à seguinte definição:

Seja $C \subset \mathscr{\mathscr { C }}_{A}(\sigma, t), E \subset \mathscr{E}_{A}(\sigma, t), S \subset \mathscr{Q}_{A}(\sigma, t), r \in S$, uma relação $r: \mathscr{E}_{A}(\sigma, t) \rightarrow \mathscr{C}_{A}(\sigma, t)$ pertencente à A-Estrutura de um sistema de transporte $\sigma \in \Sigma$. Seja ainda, $r_{1}$ uma relação sujeito-objeto definida por $r_{1}: \mathscr{E}_{A}(\sigma, t) \rightarrow \mathscr{E}_{A}(\sigma, t)$, e $x_{1}, x_{2} \in E$. 
Diz-se que a coisa $x_{2}$ tem mobilidade sob o sistema de transporte $\sigma$, ou " $M_{\sigma} x_{2}$ ", se, e somente se, o sistema é acessível tanto a $x_{1}$ (Sujeito de transporte correspondente a $x_{2}$ ) quanto a $x_{2}$ (objeto de transporte correspondente a $x_{1}$ ). Isso pode ser formalizado da seguinte forma:

$$
M_{\sigma} x_{2} \leftrightarrow\left(\left\langle x_{1}, x_{2}\right\rangle \in r_{1} \wedge A_{\sigma} x_{1} \wedge A_{\sigma} x_{2}\right)
$$

Continuando o exemplo da seção anterior. Seja $x_{1}$ uma indústria de alimentos, $y$ uma empresa de transportes de cargas (que compõe o sistema de transporte de cargas), e $r$ uma relação " $x$ contrata e paga $y$ ". Adicionemos ao caso, $r_{1}$ uma relação " $x_{1}$ precisa que $x_{2}$ seja entregue no centro consumidor", e $x_{2}$ uma carga de carne bovina. Dizemos que, nesse caso, a carga de carne bovina tem mobilidade sob o sistema de transporte considerado se, e somente se, existe uma intenção ou necessidade de transporte, se for possível que a empresa contrate e pague o valor cobrado pela empresa transportadora (ex: existem escritórios da empresa na localidade, ou meios de comunicação para estabelecimento do contato, a indústria dispõe de recursos financeiros suficientes etc) e se a carne bovina puder ser transportada pela empresa (ex: carros frigoríficos, se existirem estradas, pessoal capacitado etc).

No exemplo utilizado não foi adotado um caso curioso: uma pessoa que deseja sair de sua casa para realizar compras numa loja, considerando que irá a pé. Nesse caso: $x_{1}$ seria a pessoa (o ente social, que deseja participar da atividade); $y$ seria qualquer elemento de um conjunto constituído pelo sistema locomotor de seu corpo, ruas, escadas, passarelas, dentre outros; $x_{2}$ seria o próprio corpo. Esse exercício é meramente conceitual, e aqui se reconhece ser, aparentemente, muito estranho proceder a tal tipo de análise. Contudo, para melhor compreender os sistemas de transporte e a questão da mobilidade é uma abordagem fecunda, pois põe novos elementos analíticos e novas relações em evidência (passando a reconhecer o corpo humano como um tipo de veículo que pode ser utilizado num sistema de transporte), trazendo novos aspectos a serem incorporados no desenho desse tipo de sistemas e seus componentes. O pedestre deixaria de ser um mero ente acessório, e a teoria e técnica de desenho de vias e outros artefatos passaria a incorporar, de forma mais orgânica, as propriedades desse tipo de "veículo", por exemplo.

\section{CONCLUSÕES E INDICATIVOS DE ESTUDOS FUTUROS}

Este trabalho buscou contornar um problema comum, mas ainda não diretamente abordado, na pesquisa sobre Transporte e Mobilidade: a imprecisão das definições de acessibilidade e mobilidade que não apenas provocam confusão - pela hiperinflação dos conceitos - entre os pesquisadores, como também, em muitos casos, induzem a equívocos - por exemplo, estagnação teórico-metodológica muitas vezes mascarada pela crença de uma real mudança qualitativa no planejamento urbano, de transporte e mobilidade, pela simples invocação do termo 'mobilidade'; ou, ainda, a associação da própria noção de mobilidade ao número de viagens realizadas que, por sua vez, conduz ao equívoco do "quanto mais melhor".

Como forma de contornar, partiu-se da teoria de Mario Bunge e definição de "Transporte" para produzir uma definição formalizada para os mencionados termos. Apesar das dificuldades iniciais que a notação possa apresentar para aqueles que não estão acostumados, é interessante perceber que foi possível obter uma acepção inequívoca, geral 
e passível de especialização dentro de limites consistentes. Ou seja, é possível estabelecer contextos de aplicação através da simples restrição do âmbito da A-Estrutura, AComposição e A-Ambiente - ver definição de acessibilidade no item 5.1. Outro ponto de destaque é que as definições apresentadas mantém consistência com a origem etimológica dos termos, e neste sentido, atendem ao requisito das definições de guardarem coerência com o senso comum.

Além disso, vale reforçar os seguintes pontos: (i) as definições apresentadas abrem novas perspectivas analíticas derivadas da própria teoria que lhes serviu de base: o modelo de sistema de Bunge; (ii) os enfoques de acessibilidade e mobilidade trazem de forma inequívoca o indivíduo para o centro da questão para efetiva compreensão e previsão. Ou seja, a gênese do transporte, a própria acessibilidade ( $e$, por consequência, a mobilidade) dependem de características dos sujeitos demandantes do transporte e, claro, de propriedades dos sistemas de transporte/mobilidade e não de um contexto "generalizado" como usualmente se desenvolve nos planos de transporte e mobilidade; (iii) as definições propostas sugerem mudança de enfoque no planejamento de transporte/mobilidade, reforçando iniciativas como a TDM - Transport Demand Management (OHTA, 1998); (iv) a teoria de base oferece recursos consistentes para a aplicação de modelagem de redes sociais (Nooy et al, 2005) para a própria modelagem de sistemas de transporte/mobilidade, ou mesmo modelagem "ontológica" com ferramentas como o Protégé (Gennari et al, 2002).

Espera-se que as definições oferecidas possam guiar futuros estudos sobre o tema, inclusive diminuindo a confusão terminológica da área. Além disso, reforça-se a necessidade de se separar a definição do conceito acessibilidade e mobilidade de suas condições materiais de realização, visto que essas últimas, em grande parte dos casos, dependem de uma decisão de âmbito político muito mais que técnico (por exemplo, qual o limite de renda que é adequado estabelecer para julgar alguém como privado de acesso?).

Por fim, para continuidade dos estudos, sugere-se:

- a aplicação das definições apresentadas, inclusive a de sistema concreto de Bunge, para a modelagem de um caso exemplar de sistema de transporte/mobilidade utilizando tanto as ferramentas Pajek (Nooy et al, 2005) quanto o Protégé (Gennari et al, 2002);

- estudo sobre as condições materiais para a asserir se o sistema de transporte/mobilidade é, ou não, acessível, e/ou se determinado indivíduo, ou objeto, é móvel (ou, possui mobilidade);

- revisão das formas de planejamento, tanto do ponto de vista teórico quanto metodológico, para abordagem das questões de transporte e mobilidade.

\section{REFERÊNCIAS BIBLIOGRÁFICAS}

ANDLER, Daniel; FAGOT-LARGEAULT, Anne; SAINT-SERNIN, Bertrand. FILOSOFIA DA CIÊNCIA. Volume II. Atlântica, Rio de Janeiro, 2005.

BRASIL. LEI NO 10.098, DE 19 DE DEZEMBRO DE 2000. Estabelece normas gerais e critérios básicos para a promoção da acessibilidade das pessoas portadoras de deficiência ou com mobilidade reduzida, e dá outras providências. Presidência da República, Brasília, 2000.

BRASIL. LEI N. 12.587, DE 3 DE JANEIRO DE 2012. Institui as Diretrizes da Política Nacional de Mobilidade Urbana. Presidência da República, Brasília, 2012. 
BUNGE, Mario A. TREATISE ON BASIC PHILOSOPHY - ONTOLOGY I: THE FURNITURE OF THE WORLD. D. Reidel Publishing Company, Dordrecht, 1977.

BUNGE, Mario A. TREATISE ON BASIC PHILOSOPHY - ONTOLOGY II: A WORLD OF SYSTEMS. D. Reidel Publishing Company, Dordrecht, 1979.

COX, K. R. MAN, LOCATION AND BEHAVIOR: AN INTRODUCTION TO HUMAN GEOGRAPHY. Wiley, New York, 1972.

EUROPEAN ENVIRONMENT AGENCY - EEA. MULTILINGUAL ENVIRONMENTAL GLOSSARY. Disponível em: http://glossary.eea.eu.int/EEAGlossary/M/mobility, 2006.

FORTHERINGHAM, A. S; BRUNSDON, C.; CHARLTON, M. QUANTITATIVE GEOGRAPHY: PERSPECTIVES ON SPATIAL DATA ANALYSIS. Sage Publications, London. 2002.

GENNARI, J.; FERGERSON, R.; GROSSO, W. E.; CRUBEZY, M.; ERIKSSON, H.; NOY, N. F.; TU, S. W.; MUSEN, M. A. THE EVOLUTION OF PROTÉGÉ: AN ENVIRONMENT FOR KNOWLEDGEBASED SYSTEMS DEVELOPMENT. Stanford Center for Biomedical Informatics Research, Stanford, 2002.

GIRLE, R. POSSIBLE WORLDS. McGill-Queen's, Montreal\&Kingston., 2003.

HOUAISS, A; VILLAR, M. de S.; FRANCO, F. M. de. DICIONÁRIO HOUAISS DA LÍNGUA PORTUGUESA. Objetiva, Rio de Janeiro, 2001.

HUGHES, G. E.; CRESSWELL, M. J. A NEW INTRODUCTION TO MODAL LOGIC. Routledge: London \& New York, 1996.

MAGALHÃES, Marcos T. Q. FUNDAMENTOS PARA A PESOUISA EM TRANSPORTES: REFLEXÕES FILOSÓFICAS E CONTRIBUIÇÃO DA ONTOLOGIA DE BUNGE. Tese de Doutorado em Transportes. Universidade de Brasília, Brasília, 2010.

MINISTÉRIO DAS CIDADES. POLÍTICA NACIONAL DE MOBILIDADE URBANA SUSTENTÁVEL. MCidades, Brasília, 2004.

MORLOCK, Edward K. INTRODUCTION TO TRANSPORTATION ENGINEERING AND PLANNING. McGraw-Hill, Tóquio, 1978.

NOOY, Wouter; MRVAR, Andrej; BATAGELJ, Vladimir. EXPLORATORY NETWORK ANALYSIS WITH PAJEK. Cambridge Press, Cambridge, 2005.

OHTA, Katsutoshi. TDM MEASURES TOWARDS SUSTAINABLE MOBILITY. IATSS Research, Vol.22, No. 1, p.6-13, 1998.

ORTÚZAR, J.; WILLUMSEN, L. G. MODELING TRANSPORT. Wiley, Chichester, 1997.

PAPACOSTAS, C. S.; PREVEDOUROS, P. D. TRANSPORTATION ENGINEERING AND PLANNING. Prentice Hall, Englewood Cliffs, 1993.

TAAFFE, E. J.; GAUTHIER, H. L.; O’KELLY, M. E. GEOGRAPHY OF TRANSPORTATION. PrenticeHall, New Jersey, 1996.

VUCHIC, V. TRANSPORTATION FOR LIVABLE CITIES. Rutgers, New Jersey, 2000. 